\title{
Компьютерное моделирование кинетического массопереноса и поведение многокомпонентных концентрационных волн в би-функциональных моделях сорбентов - нано-композитов
}

\author{
Калиничев А.И. \\ ФГБУН Институт физической химии и электрохимии имени А.Н. Фрумкина, Москва
}

Поступила в редакцию 30.10.2018 г.

DOI: https://doi.org/10.17308/sorpchrom.2018.18/590

\begin{abstract}
Представлены результаты теоретического численного компьютерного исследования кинетики Много(n=6)-компонентного Массо-Переноса (ММП) в современных комбинированных сорбционных материалах-НаноКомпозитах (НК). Продемонстрированы визуально примеры Много(n=6)компонентных концентрационных $\left\{\mathrm{X}_{\mathrm{n}}(L, \mathrm{~T})\right\}$-волн, распространяющихся в планарных HK L-матрицах селективных би-функииональных НК, где внутри ИО-матрицы расположены агломераты “НаноЧастиц (а именно $\mathrm{HЧ}^{0}\left(\right.$ Металл$\left.^{0}\right)$, образующие внутри активные нуль зарядные (“" $\mathrm{H}^{0}\left(\mathrm{Me}^{0}\right)$-наносайты”). Агломераты НЧ $\left(\mathrm{Me}^{0}\right)$ обычно внедряют в изучаемую, результирующую сорбционную НК(ИО)-матрицу, образованную в результате предварительного синтеза НК.

Применение разработанного здесь современного компьютерного моделирования ММП НК сорбционной кинетики основано на на принципах термодинамики неравновесных процессов, таких как феноменологический многокомпонентный баланс масс, выраженный n-уравнениями (в частных производных), охарактеризованных термодинамическим, фундаментальным интегральным параметром: ${ }^{\text {new }} \mathrm{k}^{(2)}(6)$-“вариантностью”, где k-параметр описывает термодинамическую “степень свободы” $\left(n=\mathrm{k}^{(2)}=6\right)$ для рассматриваемой много(n)-компонентной ММП-кинетической сорбционной НК-системы. Рассматривается волновая (W)-концепция термодинамики неравновесных процессов, а именно взаимодействие много-компонентных распространяющихся $\left\{\mathrm{X}_{\mathrm{n}}(L, T)\right\}$-концентрационных волн n-компонентов в их распространении через планарную HK L-мембрану. Распространение взаимодействующих и распространяющихся $\left\{\mathrm{X}_{\mathrm{n}}(L, \mathrm{~T})\right\}$-концентрационных волн $\mathrm{n}$-компонентов демонстрируется на основе авторской визуализации после обработки результатов расчетов при моделировании с помощью создаваемых на компьютере Научных Компьютерных Анимаций, а именно многоцветных НКА видео файлов.

Ключевые слова: НаноКомпозиты(НК), "НЧ-нано-сайты“", взаимодействие концентрационных волн, селективность, сорбционная НК кинетика, би-функциональная НК Модель, вытеснение волн (ВВ), компьютерное моделирование, визуализация волн.
\end{abstract}

\section{Computer Simulation of the kimetic mass transfer and multicomponent concentration waves behavior in bi-functional models for sorbents-nano-composites}

\author{
Kalinitchev A.I. \\ Frumkin Institutde of Physical Chemistry and Electrochemistry, Russian Academy of Sciences, Moscow \\ There is realized the theoretical investigation of the Multi(n=6)-component Mass Transfer (MMT) \\ kinetic process inside the modern combined sorption "NanoComposites (NC)" materials. The NC examples \\ are represented in the manuscript by the bi-functional Selective "Metal ${ }^{0}$-Ion Exchangers" NC matrix where
}


the inner active centers - "NP nanosites" conditioned as "the " $\left(\mathrm{Me}^{0}\right)$ Nano-Particles agglomerates" which are imbedded into the IEx matrix during the preliminary synthesis of the combined NC matrix. There is considered the MMT NC kinetic process across (inside) the bi-functional NC planar L-matrix via the author's computerized numerical modelling by the mathematical solution of the mass balance $\mathrm{n}(6)$-Eqns.

The elaborated computerized simulation approach for the MMT NC kinetic process is based on the foundations of the irreversible thermodynamics including the phenomenological multi(n)-component $(n=6)$ mass balance $n$-Eqns. (in partial differenthials) characterizing by the integral parameter ${ }^{\text {new }} \mathrm{k}^{(2)}$-variance where the thermodynamic fundamental integral k-parameter describes "the number of the degree of freedom" $\left(n=k^{(2)}=6\right)$ of the MMT combined bi-functional NC system considered. There is considered the wave concept $\left(\mathrm{W}^{+}\right)$of the thermodynamic of the irreversible process with the propagating mode of the interacting and multi-component $\left\{\mathrm{X}_{\mathrm{n}}(\mathrm{L}, \mathrm{T})\right\}$-concentration waves for the $\mathrm{n}$-components spreading through the planar NC Lmembrane. The propagation of the interacting and propagating $\left\{X_{n}(L, T)\right\}$-concentration waves is demonstrating on the basis of the author's visualization after the computerized treatment of the results of the modeling with the creation of the Sci Computerised Animations, namely by the creation of the multi-color SCAanimated video-files.

Keywords: NanoComposites(NC), "NP-nano-sites", concentration waves interaction, selectivity, sorption NC kinetics, bi-functional, NC Models, chromatographic Displacement Development (DD), computer simulation, visualization

\section{Введение}

Ряд дополнительных фундаментальных характеристик упомянутой неравновесной термодинамики также включены в рассмотрение, а именно Закон Действующих Масс (ЗДМ $\mathrm{S}_{\mathrm{S}}$; ключевая волновая феноменологическая (W)-концепция распространяющихся (во времени,T и пространстве,L) много(n=6)-компонентных $\left\{\mathrm{X}_{\mathrm{n}}(L, \mathrm{~T})\right\}$-концентрационных волн (распределений) в НК мембране $(\mathrm{n}=1,2, . .6)$; механизм преобразования масс “сток-осточник”, определяемый ЗДМ $\mathrm{S}$ соотношениями; фундаментальные уравнения Нернста-Планка для $\mathrm{J}_{\mathrm{n}}$-Потоков масс; соотношение электро-нейтральности и ряд других [1].

Рассматриваемое далее теоретическое компьютерное моделирование основано на двух видах различных би-функциональных $\mathrm{k}^{(1,2)}$-НК Моделей, а именно описываемая здесь новая $\left({ }^{\mathrm{new}} \mathrm{k}^{(2)}\right)$ )-НК Модель и предыдущая $\left({ }^{\mathrm{prev}} \mathrm{k}^{(1)}\right)$-НК Модель [2], которые отличаются принципиально различными значениями термодинамических kвеличин “степеней свободы”, где ${ }^{\text {new }} \mathrm{k}^{(2)}(6)>{ }^{\mathrm{prev}} \mathrm{k}^{(1)}(5)$ [2].

Современное компьютерное моделирование, основанное на бифункииональной ${ }^{\text {new }} \mathrm{k}^{(2)}$-НК Модели, приносит новые финальные результаты, относительно взаимодействия принципиальных сорбционных $\left\{\mathrm{X}_{n}\right\}-$ много(n=6)компонентных концентрационных волн, , которые описывают ММП НК кинетический процесс на основе компьютерного расчета и фундаментальной феноменологической волновой $(\mathrm{W})$-концепции[2], упоминаемой ранее.

Bce $\mathrm{n}(6)$-компоненты рассматриваемой здесь $\left\{\mathrm{X}_{\mathrm{n}}\right\}$-концентрационной композиции $\left\{{ }_{\mathrm{m} 1,2}\left(\mathrm{R}^{0} \mathrm{p}\right)^{+} ; 3,4 \mathrm{p}^{+} ; \mathrm{j}_{5}^{-} ;{ }_{6} \mathrm{R}^{0}\right\}$-участвуют в ММП НК кинетическом сорбционном процесса внутри би-функциональной HK L-матрицы [2] (с двумя со-существующими ММП маршрутами, а именно- $\left\{\right.$ селективным, I и I,много-диффузионным, $\left.\mathrm{D}_{3-5}\right\}-$ рис.1.( $a-c)$. На принципиальных схемах рис.П(a-c) изображены все составляющие современной новой би-функциональной ММП расширенной ${ }^{\text {new }} \mathrm{k}^{(2)}(6)-Н К$ Модели, включающей принципиальные парные “сорбат $(3,4 \mathrm{p})$-сорбент $\left(\mathrm{m}_{1,2}\right)$ "- $\left\{3,4 \mathrm{p} \mathrm{x}_{1,2}\right\}$ компоненты.

Расширенная ММП НК сорбционная система, описываемая упомянутой бифункциональной ${ }^{\text {new }}{ }^{(2)}(6)$-НК Моделью, включает четыре парных $\left\{2_{\mathrm{p}}{ }^{\mathrm{X}} 2_{\mathrm{m}}\right\}=\left\{\mathrm{l}_{3,4} \mathrm{p}^{\mathrm{X}} \mathrm{m}_{1,2}\right\}$ принципиальных сорбирующихся-компонент в $\left(\mathrm{I}_{1,2}\right)$ (рис. $\left.1 b, c\right)$ с их участием в стадиях «сорбция $(\mathrm{I} a-)$-десорбция $(-\mathrm{I} d)$ для $\left(\mathrm{I}_{1,2}\right)-3 Д \mathrm{M}_{\mathrm{S}}$ реакций (рис. $\left.1 b, c\right)$. Детали ав- 
торской новой ММП ${ }^{\text {new }} \mathrm{k}^{(2)}-\left\{2_{\mathrm{p}}{ }^{\mathrm{x}} 2_{\mathrm{m}}\right\}$ НК Модели иллюстрированы концептуальными визуальными схемами на рис. 1 a-c.

Участие пяти целевых (принципиальных) $\left\{\mathrm{m}_{1,2} ;{ }_{(3,4)} \mathrm{p} ;{ }_{6} \mathrm{R}^{0}\right\}$-компонентов, а именно: четырех парных $\left\{2_{\mathrm{p}} \mathrm{X} 2_{\mathrm{m}}\right\}$-принципиалов (см. выше) совместно с последним и решающим $\left(\mathrm{k}=6_{6} \mathrm{R}^{0}\right)$-принципиальным, $6^{\mathrm{M}}$-компонентом-сорбентом. Роль НКсорбента (на активных центрах: $\mathrm{HЧ}^{0}$ - “наносайтах” которого осуществляется сорбция в виде $\mathrm{m}_{1,2} \equiv_{\mathrm{m} 1,2}\left(\mathrm{R}^{0} \mathrm{p}\right)^{+}$-“комплексов” (рис. $\left.1 b, c\right)$ играет нуль зарядный $6^{\breve{1}}$ компонент$k=6 \mathbf{R}^{0}$.

Этот ключевой ${ }_{k} \mathrm{R}^{0}$ компонент-(названный здесь "наносайты") с переменной концентрацией-[ $\left.{ }_{k} \mathrm{R}^{0}\right]$, играющий роль сорбента (с “активными центрами-НЧ ${ }^{0}$ агломератами") фундаментально важен для исследования интерференции “необычных" $\left\{\mathrm{X}_{1,2,6}(L, \mathrm{~T})\right\}$-распространяющихся в НК концентрационных волн, рассчитанных в результате примененного здесь современного компьютерного моделирования.

В компьютерном моделировании кинетического ММП процесса через планарную HK L-мембрану (рис. $1 a$ ) реализуется переменная $\left[{ }_{\mathrm{k}} \mathrm{R}^{0}\right]-$-концентрация в принципиальной сорбционной $\mathrm{X}_{\mathrm{k}=6}(L, \mathrm{~T})$-концентрационной волне. Физический смысл возникновения и распространения такой “интегральной” ${ }_{k} R^{0}$-волны (рис. $2 \mathrm{~A}, \mathrm{~B})$ понятен - это результат изменения концентраций принципиальных состав-

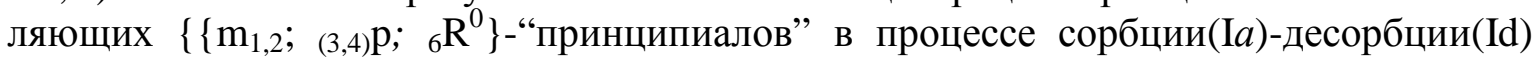
ЗДМ $\mathrm{M}_{\mathrm{S}}$ реакций $\left(\mathrm{I}_{1,2}\right)$ (см.рис электронейтральность в ,би-функциональной комбинированной ММП НК сорбционной системе, при наличии $\left\{\left(\mathrm{I}_{1,2}\right)\right.$-селективной сорбции и II- $\left\{\mathrm{D}_{3-5},\right\}$, много-диффузионности $\}$.

Интерференция необычных $\left\{\mathrm{X}_{1,2,6}(L, \mathrm{~T})\right\}$-траспространяющихся концентрационных волн (хотя их диффузионность отсутствует, $\mathrm{D}_{1,2,6}=0$ ) приносит, (в частности во фронтальном режиме) хроматографический Вытеснительный Режим (ВР) в поведении двух интерферирующих $\mathrm{X}_{1,2}(L, \mathrm{~T})$-волн: $\mathrm{X}_{1}$-вытеснителя, и $\mathrm{X}_{2}(L, \mathrm{~T})$ вытесняемой концентрационных волн.. $1 a-c)$. На рис. 2(A,B, $\mathrm{T}^{1-3} ;$;ривая,6), представляющем результат “численного эксперимента"-моделирования, этот принципиально важный новый результат визуально подтверждается наличием распространяющейся (во времени $\left(\mathrm{T}^{1-3}\right)$ и пространстве) “интегральной” принципиальной сорбционной $\mathrm{X}_{\mathrm{k}=6}\left(L, \mathrm{~T}^{1-3}\right)$-волны (рис.2А,вверxy;B,внизу, интегральные кривые $6, \mathrm{~T}^{1-3}$ серии).

Фундаментальный и ключевой ${ }_{6} \mathrm{R}^{0}$-принципиальный компонент характеризуется различными $\left[{ }_{6} \mathrm{R}^{0}\right]$-концентрацями, и определен как "НЧ ${ }^{0}$-наносайты". Кардинальный и фиксированный компонент ${ }_{6} \mathrm{R}^{0}$-(под наибольшим $6^{\mathrm{M}}$-номером (слева от $\mathrm{R}^{0}$ ) в ММП НК системе введен здесь, (а ранее в авторских убликациях, как ${ }_{5} \mathrm{R}^{0}$ [2]) намеренно при решении теоретической задачи ММП НК кинетики сорбции в данной $\left({ }_{6} \mathrm{R}^{0}\right)$ и предыдущих $\left({ }_{5} \mathrm{R}^{0}\right)$ публикациях [2].

Кроме того, в дополнение к селективному ФММП маршруту включен в рассмотрение второй ММП $\left\{\mathrm{D}_{3-5}\right\}$-много-Диффузионный, 四 ММП маршрут для двух диффундирующих (3,4) $\mathrm{p}^{+}$-сорбат-принципиалов с введением в рассмотрение диффундирующих ( $\left.\mathrm{D}_{5}>0\right) \mathrm{j}_{5}^{-}$-ко-ионов. Соответствующий поток $\mathrm{j}_{5}$-коионов стремится поддерживать электронейтральность в би-функциональной комбинированной ММП НК сорбционной системе, при наличии $\left\{\left(\mathrm{I}_{1,2}\right)\right.$-селективной сорбции и $\mathrm{II}\left\{\mathrm{D}_{3-5},\right\}$, многодиффузионности.

Интерференция необычных $\left\{\mathrm{X}_{1,2,6}(L, \mathrm{~T})\right\}$-траспространяющихся концентрационных волн (хотя их диффузионность отсутствует, $\mathrm{D}_{1,2,6}=0$ ) приносит, (в частности во фронтальном режиме) хроматографический Вытеснительный Режим (ВР) в поведении двух интерферирующих $\mathrm{X}_{1,2}(L, \mathrm{~T})$-волн: $\mathrm{X}_{1}$-вытеснителя, и $\mathrm{X}_{2}(L, \mathrm{~T})$ вытесняемой концентрационных волн. Для ММП НК сорбционной кинетики визуально продемонстрирована (посредством созданных расчетных много-цветных ани-

Калиничев А.И. / Сорбционные и хроматографические процессы. 2018. Т. 18. № 5 
маций или “остановленных видео-изображений”, см. рис.2), что причина атипичного (и необычного) поведения $\left\{\mathrm{X}_{1,2,6}(L, \mathrm{~T})\right\}$-концентрационных волн состоит в комбинированном свойстве, выражаемом би-функциольнальностью НК матриц в комбинации двух \{Селективный, I I I, \{ Диффузионный $\}$ - ММП со-маршрутов (рис.1a-c).

\section{Современная Расширенная би-функциональная $\mathbf{k}^{(2)}(6)-Н К$ Модель в Многокомпонентной НК Кинетике Сорбции}

В данной работе современное компьютерное моделирование кинетики Много $(\mathrm{n}=6)$-компонентного Массо-Переноса (ММП) основано на принципах термодинамики неравновесных процессов [1,2] включая приближенное математическое компьютерное решение феноменологических уравнений многокомпонентного ММПбаланса масс, выраженного n-уравнениями (в частных производных), включая современную ${ }^{\text {new }}{ }^{(2)}(6)-\left\{2{ }_{\mathrm{p}}{ }_{2}{ }_{\mathrm{m}}\right\}$ (см. выше) НК Модель для би-функиональной ММП НК системы в комбинации с введением двух упомянутых (迎)-ММП со-маршрутов (рис.1a-c).

Много(n=6)-компонентный ключевой механизм “сток-источник” вдоль селективного Џмаршрута в НК-матрице (рис. 1a-c) [2] для ММП селективного円маршрута (с двумя $\left\{\mathrm{K}_{\mathrm{S}}{ }^{1} ; \mathrm{K}_{\mathrm{S}}{ }^{2}\right\}$-параметрами селективности в $\left(\mathrm{I}_{1,2}\right)$ и $\left(\mathrm{I}_{\mathrm{K}} \mathrm{K}_{1.2}\right)$-3ДМ соотношениях, рис. 1 ( $a$-c). Эти параметры введены для дальнейшего теоретического рассмотрения и моделирования сорбционного процесса для четырех $\left\{\mathrm{m}_{1,2} \&_{3,4} \mathrm{p}\right\}$ принципиальных парных $\left\{1,2 \mathrm{p}^{\mathrm{X}} \mathrm{m}_{1,2}\right\}=\left\{2 \mathrm{p}^{\mathrm{x}} 2 \mathrm{~m}\right\}$-сорбирующихся компонентов (см. рис. $1 a$-с). Детальное описание иллюстрируется принципиальными схемами здесь на рис. $1 a, b, c$ и ранее в работах [2-6]

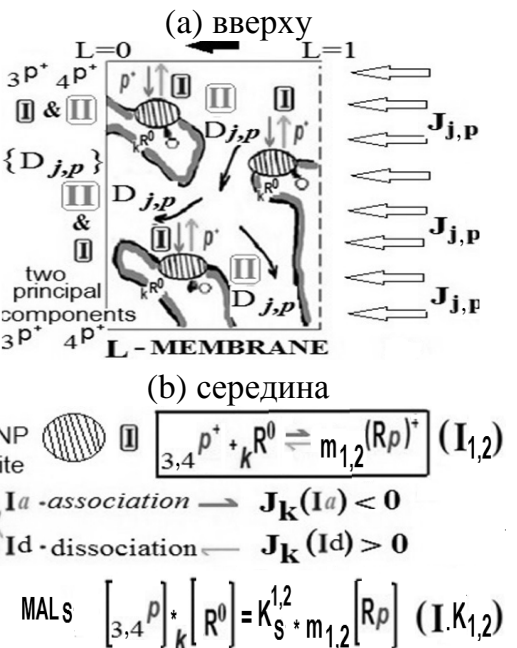

(c) внизу

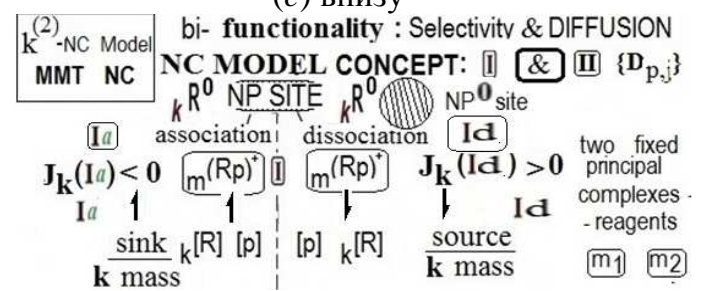

Рис. 1 (а) вверху), $b\left(\right.$ середина),c(внизу).Авторская “расширенная“ ${ }^{\text {new }} \mathrm{k}^{(2)}(6)-\left\{2_{\mathrm{p}}{ }^{\mathrm{x}}{ }_{\mathrm{m}}\right\} \mathrm{HK}$ Модель. Принципиальные схемы, и соотношения, включающие селективный (I, $\left.\mathrm{I}_{1,2}\right)$-ММП маршрут $\mathrm{I} a-c) ;\left\{\mathrm{K}_{\mathrm{S}}{ }^{1} ; \mathrm{K}_{\mathrm{S}}{ }^{2}\right\}$-коэф. Селективности; “сток-источник” в механизме "трансформа-

ция масс" $(a, \mathrm{c}) ; \mathrm{J}_{\mathrm{k}, \mathrm{m}, \mathrm{p}}$-Потоки масс $(a . b)$; ключевой $\mathrm{k}=6 \mathrm{R}^{0}-6^{\text {й }}$ (“нано-сайты”-агломераты НЧ, штрих; ${ }_{6} \mathrm{R}^{0}$ - нуль зарядный $6^{\breve{и ̆}}$-компонент, $a$-c); $3 Д \mathrm{M}_{\mathrm{S}}\left(\mathrm{I}_{\mathrm{K}}, 2\right)$-соотношения селективности для $\mathrm{k}=6 \mathrm{R}^{0}(b)$; парные $\left\{{ }_{(3,4)} \mathrm{p}^{\mathrm{x}} \mathrm{m}_{1,2}\right\}$-компоненты “сорбат-сорбент”, (a) I- $\mathrm{N}$ ного- $\left\{\mathrm{D}_{3-5}\right\}$ -

Диффузионный со-маршрут. 
В данной работе реализовано расширенное современное теоретическое компьютерное исследование много $(\mathrm{n}=6)$-компонентного сорбционного кинетического процесса Массо-Переноса (ММП) в НК-би-функциональной матрице на основе упоминавшейся принципиально модернизированной ${ }^{\mathrm{new}} \mathrm{k}^{(2)}-\left\{2_{\mathrm{p}}{ }^{\mathrm{x}} 2_{\mathrm{m}}\right\}$ НК Модели (Рис.1 $a$ c ), обладающей “расширенной” new $\mathrm{k}^{(2)}(6)$-вариантностью, где, очевидно для новой вариантности ${ }^{\text {new }} \mathrm{k}^{(2)}(6)={ }^{\text {prev }} \mathrm{k}^{(1)}(5)+1$.

Приводимое здесь ${ }^{\text {new }} k^{(2)}-\left\{2{ }_{p}{ }^{\mathrm{x}} 2_{m}\right\}$-обозначение для ${ }^{\mathrm{new}} \mathrm{k}^{(2)}$-НК Модели подчеркивает характеристики здесь усоверщенствованной $\mathrm{n}(6)$-компонентной ММП сорбционной НК системы $\mathrm{c}^{\text {new }} \mathrm{k}^{(2)}(6)$-вариантностью, включая две пары $\left\{\left({ }_{3} \mathrm{p}^{\prime} \mathrm{m}_{1}\right) \&\left({ }_{4} \mathrm{p}^{\prime} \mathrm{m}_{2}\right\}\right.$ принципиальных “сорбат $(3,4 \mathrm{p}) /$ сорбент $\left(\mathrm{m}_{1,2}\right)$ ”-компонентов. Два диффундирующих целевых-принципиальных $(3,4) \mathrm{p}^{+}$-компонента-“сорбата”, и соответственно сорбированных целевых (принципиальных) $\mathrm{m}(1,2)(\mathrm{R} p)^{+}$-“комплекса" на ${ }_{6} \mathrm{R}^{0}$-“наносайтах” составляют участников двух ЗДМ ${ }_{\mathrm{S}}\left(\mathrm{I}_{1,2}\right)$-реакций (рис. $1 b$ ) совместно с соответствующими парными ЗДМ ${ }_{\mathrm{S}}\left(\mathrm{I}_{\mathrm{K}} \mathrm{K}_{1,2}\right)$-соотношениями (рис. $\left.1 b\right)$.

Эти (I.K $\left.{ }_{1,2}\right)-3 Д M_{S}$ селективные соотношения включают два параметра $\left\{\mathrm{K}_{\mathrm{S}}{ }^{1} ; \mathrm{K}_{\mathrm{S}}{ }^{2}\right\}$-Селективности для двух $\mathrm{m}(1,2)(\mathrm{R} p)^{+}$-сорбированных (и фиксированных, $\left.\mathrm{D}_{1,2}=0\right)$ компонент-участников в $3 Д \mathrm{M}_{\mathrm{S}}\left(\mathrm{I}_{\mathrm{K}} \mathrm{K}_{1,2}\right)$-селективных соотношениях (см. принципиальные схемы на рис. $1 a-c$. Так что упомянутая расширенная и “продвинутая" ${ }^{\text {new }} \mathrm{k}^{(2)}(6)$-HК Model включает четыре $\left\{{ }_{(3,4)} \mathrm{p} / \mathrm{m}_{1,2}\right\}$ - сорбат $(3,4 \mathrm{p}) /$ сорбент $\left(\mathrm{m}_{1,2}\right)$ принципиала, участвующих в двух селективных парных $\mathrm{MMП}\left(\mathrm{I}_{1,2}\right)$-реакциях: сорбции $(\mathrm{I} a-)$-десорбции $(-\mathrm{I} d)$ (рис. $1 b, c)$ совместно с соответствующими селективными парными ЗДМ $\mathrm{S}\left(\mathrm{I}_{1,2}\right)$-соотношениями (рис. $\left.1 b\right)$.

Таким образом, рассматриваемый сорбционный процесс в бuфункциональных НК матрицах включает пять принципиальных $\left\{(\mathrm{m}(1,2) \mathrm{Rp})^{+} ; 3,4 \mathrm{p}^{+}\right.$; ${ }_{6} \mathrm{R}^{0}$ \}-компонентов-участников для двух парных, сорбционных $\mathrm{MM \Pi}\left(\mathrm{I}_{1,2}\right)$ селективных реакций (см. рис. 1 - $-c$ ) включающих два парных $\left\{(3,4) \mathrm{p} / \mathrm{m}_{1,2}\right\}=\left\{2_{\mathrm{p}}{ }^{\mathrm{x}} 2_{\mathrm{m}}\right\}$ принципиалов, а именно два $3,4 \mathrm{p}^{+}$-сорбат-компонента и два $\mathrm{m}_{1,2}$-сорбированных недиффундирующих (где $\left.\mathrm{D}_{1,2}=0\right), \mathrm{m1,2}(\mathrm{R} p)^{+}$-“комплекса" а также определенный ранее ключевой принципиальный ${ }_{\mathrm{k}} \mathrm{R}^{0}$-компонент-участник в $\left(\mathrm{I}_{1,2}\right)($ рис. $1 a, b)$.

MМП НК сорбционный процесс реализуется через планарный HK L-слоймембрану $(0 \leq \mathrm{L} \leq 1)$, справа $\left(\mathrm{L}_{0}=1\right)$ налево $(\mathrm{L} l=0)$ на рис. $1 a$. ММП НК сорбционный процесс кинетики включает два маршрута, а именно \{селективный, I \& II $\left\{\mathrm{D}_{3-5}-\right.$ много-диффузионный ММП со-маршрут с изображением на принципиальных визуальных схемах на рис.1( $a$-c).

В данной статье рассмотрено теоретически ММП НК сорбционный кинетический процесс внутри планарной HK L-матрицы посредством разработанного современного численного компьютерного моделирования на основе компьютеризированной и усовершенствованной би-функциональной ${ }^{\text {new }} \mathrm{k}^{(2)}-\left\{2_{\mathrm{p}}{ }^{\mathrm{x}} 2_{\mathrm{m}}\right\}$ НК Модели, описываемой в данном разделе.

В итоге Рисунок 1a-c показывает визуальные схемы для НК планарных Lматриц-мембран, а,вверху), которые включают активные "NP0 ховано), образованные как агломераты нуль зарядных Нано-Частиц (НЧ $\left.{ }^{0}\right)$. Внутри Lмембраны изображены (в увеличенном масштабе, рис. $1 a, b)$ "НЧ ${ }^{0}$-нано-сайты" ( $a-c$, заштриховано) совместно с порами, в которых проходит диффузия $\left(\mathrm{D}_{3-5}\right)$ целевых сорбат $(3,4) \mathrm{p}^{+}$-компонентов с подключением движущихся $\mathrm{j}_{5}^{-}$-ко-ионов. Многодиффузионный $\left(\mathrm{D}_{3-5}\right)$ ПММП маршрут показан тонкими стрелками $(\longrightarrow)$ в порах НК L-матрицы (рис. 1a).

Маршрут ММП], определяемый двумя парными селективными 3ДМ $\mathrm{M}_{\mathrm{S}}\left(\mathrm{I}_{1,2}\right)$ реакциями и соответствующими (I.K $\left.\mathrm{K}_{1,2}\right)-3 Д \mathrm{M}_{\mathrm{S}}$-соотношениями (рис.1b) изображен вертикальными $(\uparrow \downarrow)$ стрелками вблизи "НЧํ-наносайтов" ( $\mathrm{HЧ}^{0}$ сайты- заштрихова- 
$H b l$, рис. $1 a)$. Кратко визуально-схематично представлен механизм "сток-источник" (рис.1c(Ia слева; и,Id,права)-неравенствами на рис. 1c(внизу). Кроме того, демонстрируется (светлыми стрелками $๒$ ) внешние входные $\mathrm{J}_{\mathrm{p}, \mathrm{j}}$-диффузионные потоки масс $3,4 \mathrm{p}, j_{5}{ }^{-}$-компонентов (рис. $1 a$, справа от L-мембраны).

Рцяунең 1 д демонстрирует более детально би-функииональность \{т.е. СелективностцI\& $\left\{\mathrm{D}_{3-5}\right\}$, Диффузия $\}$ комбинированной НК матрицы. Более детально представлен механизм “сток-источник” на рис. $1 b, c$. Два ЗДМ $\left(\mathrm{I}_{1,2}\right)$-соотношения и соответствующие (I.K $\left.\mathrm{K}_{1,2}\right)$-3ДМ -соотношения представлены в средней части рис. $1 b$.

Система вертикальных стрелок $(\uparrow \downarrow)$ в верхней части рис. $1 c$ в комбинации с четырьмя 3ДМ ${ }_{\mathrm{S}}\left(\mathrm{I}_{1,2}\right) \&\left(\mathrm{I}_{1,2}\right)$-соотношениями $(b)$ описывают участие принципиальных 1,2,6-компонентов в уже упомянутом механизме “сток-источник” Id, слева Id, справа).

На рис. $1 a, b$ представлен визуально пример комбинированной HK L-матрицы, содержащей внутри активные "НЧ ${ }^{0}$-наносайты”( $\mathrm{HЧ}^{0}$-штриховка, рис. $\left.1 a-c\right)$. Нуль за-

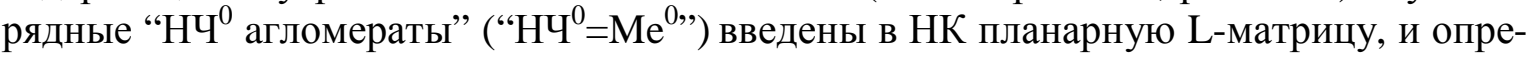
делены здесь как решающий и целенаправленно введенный в процессе создания НК Модели ${ }_{\mathrm{k}=6} \mathrm{R}^{0}-6^{\mathrm{H}}$-компонент, задаваемый переменной $\left[{ }_{6} \mathrm{R}^{0}\right]$-концентрацией (где ключевой $\mathrm{k}=6 \mathrm{R}^{0}$-принципиальный компонент-“нано-сайты” заштрихован на визуальных концептуальных диаграммах (рис. $1 a$-с).

Благодаря участию последнего $6^{\text {го }}\left({ }^{\mathrm{new}} \mathrm{k}^{(2)}=\mathrm{n}=6\right)$, фиксированного $\left(\mathrm{D}_{6}=0\right)$ и

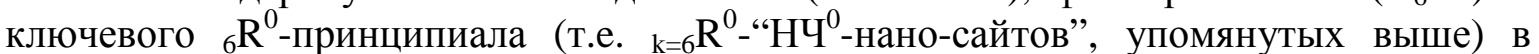
ЗДМ $\mathrm{S}$ соотношениях $\left(\mathrm{I}_{\mathrm{K}} \mathrm{K}_{1,2}\right){ }_{6} \mathrm{R}^{0}$-принципиальный компонент играет ключевую роль в процессе интерференции (взаимодействия) трех не-обычных $\left\{\mathrm{X}_{1,2,6}(L, \mathrm{~T})\right\}$ распространяющихся концентрационных волн фиксрованных 1,2,6-принципиальных компонентов $\left(\mathrm{D}_{1,2,6}=0\right)$. Особенно подчеркнем, что ключевой ${ }_{6} \mathrm{R}^{0}$-принципиал предоставляет новые фундаментальные возможности в НК кинетическом ММП процессе для созданной здесь би-функциональной ${ }^{\text {new }} \mathrm{k}^{(2)}-\mathrm{HК}$ Модели посредством введения селективной сорбционной способности и двух различных параметров $\left\{\mathrm{K}_{\mathrm{S}}{ }^{1} ; \mathrm{K}_{\mathrm{S}}{ }^{2}\right\}$ Селективности, которые включены и учитываются в двух парных $3 Д \mathrm{M}_{\mathrm{S}}\left(\mathrm{I}_{\mathrm{K}} \mathrm{K}_{1,2}\right)$ соотношениях (Рис. 1b)

Сравнивая используемые здесь две современные $\mathrm{HK}{ }^{\mathrm{prev}} \mathrm{k}^{(1)}(5) \&{ }^{\mathrm{new}} \mathrm{k}^{(2)}(6)-$ Модели можно заключить, что прежняя теоретическая ${ }^{p r e v} \mathrm{k}^{(1)}(5)-б и$-функцииональная НК Модель, рассматриваемая в более ранних работах [2-6] так же как и современная

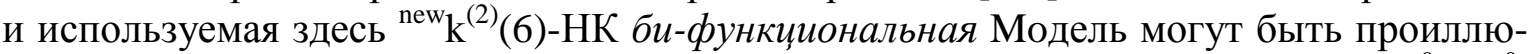
стрированы экспериментальным примером селективной НК такой как " $\mathrm{HЧ}^{0}\left(\mathrm{Me}^{0}\right.$, Металл ${ }^{0}$ в-Ионо-Обменнике(ИО)". Внутренние активные "НЧ ${ }^{0}$-нано-сайты", т.е. $\mathrm{HЧ}^{0}\left(\mathrm{Me}^{0}\right)$-агломераты $\left({ }_{6} \mathrm{R}^{0}\right)$ вводятся внутрь результирующей комбинированной ИО матрицы, полученной после предварительного экспериментального синтеза (см. далее).

Целый ряд визуальных, синтезированных в эксперименте примеров СканЭл.Микроскопии (СЭМ) би-функцииональных (“Ме ванных НК матриц демонстрируются в современной отечественной монографии [7]. Активные "Mе $\left.{ }_{(6} \mathrm{R}^{0}\right)$-наносайты" фиксированы внутри НК-матрицы в результате описанного процесса предварительного направленного экспериментального НК синтеза[7]. Теоретические аспекты синтеза НК матриц вместе с рядом практических применений обсуждены в деталях в упомянутой отечественной монографии (проф. Кравченко (ВГУ) и соавт.)[7].

Ключевой ММП НК механизм “ преобразования масс”сток-источник (на основе введения принципиального ${ }_{6} \mathrm{R}^{0}$-компонента-«наносайтов») и соответствующих внутренних $\mathrm{J}_{\mathrm{k} . \mathrm{m}, \mathrm{p}}$-массовых потоков (визуально представленных на рис. $1 a, b$ ) введен 
в компьютеризированное математическое решение феноменологических много(6)компонентных n-уравнений материального баланса[2-6].

Компьютеризированное численное моделирование с использованием nсистемы дифференциальных уравнений баланса масс (в частных производных) в результате решения приводит к многокомпонентным волновым зависимостям $\left\{\mathrm{X}_{\mathrm{n}}(L\right.$ расстояние; Т-время) $\}$. В результате получаются много-компонентные зависимости для профилей $\left\{\mathrm{X}_{\mathrm{n}}(L, \mathrm{~T})\right\}$-различных распространяющихся концентрационных волн (см. далее).

Эти концентрационные зависимости описывают распространение взаимодействующих $\left\{\mathrm{X}_{\mathrm{n}}(L, \mathrm{~T})\right\}$-волн n-компонентов-участников вдоль L-расстояния $(0 \leq \mathrm{L} \leq 1)$ с течением безразмерного Т-времени в кинетическом ММП НК сорбционном процессе. Этот процесс визуально показан компьютерными "рисунками-фреймами" далее на рис. 2(А,В; серии $\mathrm{T}^{1-3}$, верху (вариант А)-внизу(вариант В).

Три визуальных волновых $\mathrm{X}_{\mathrm{n}}\left(L, \mathrm{~T}^{1-3}\right)$-профиля для $\mathrm{T}^{1-3}$-серии (разные моменты Т- времени) изображены далее на рис. $2\left(\mathrm{~T}^{3}\right.$, слева $>\mathrm{T}^{2}$, в середине $>\mathrm{T}^{1}$, справа) и рассчитаны при компьютерном моделировании (детали даны ниже). Обсуждение далее распространяющихся много(n)-компонентных $\left\{\mathrm{X}_{\mathrm{n}}(L, \mathrm{~T}\}\right.$-концентрационных волн совместно с их сорбционным взаимодействием-интерференцией в течение компьютерного моделирования кинетического ММП НК процесса в комбинированной НК L-матрице дает в результате фундаментальное теоретическое представление рассматриваемой задачи ММП НК кинетики.

Простая прежняя ${ }^{\text {prev }}{ }^{(1)}$-НК би-функциональная Модель обладает термодинамической ${ }^{p r e v} \mathrm{k}^{(1)}=5$-вариантностью, и включает три $\left(\mathrm{m}_{1,3} \mathrm{p}_{\mathrm{k}=5} \mathrm{R}^{0}\right)$-компонентапринципиала, упомянутых выше. Это позволяет рассмотреть ММП НК кинетический волновой $(\mathrm{W})$ процесс с одной парой $\left\{1_{\mathrm{p}}{ }^{\mathrm{x}} 1_{\mathrm{m}}\right\}$ сорбирующихся (на наносайтах ${ }_{5} \mathrm{R}^{0}$ ) компонентов - $\left\{{ }_{3} \mathrm{p}^{+} / \mathrm{m}_{1}{ }^{+}\right\}$-целевых принципиалов [2-6].

В кратко обсуждаемом здесь случае ${ }^{\text {prev }} \mathrm{k}^{(1)}-\mathrm{HK}$ би-функииональной Модели много-( $\left.\mathrm{n}_{1}=5\right)$-компонентные $\left\{\mathrm{X}_{\mathrm{n} 1}(\mathrm{~L}, \mathrm{~T})\right\}$-концентрационные волны вычислялись ранее [2-6] на основе компьютерного численного решения $\mathrm{n}_{1}(5)$-уравнений ММП в частных производных. Концентрационный состав - $\left\{{ }_{\mathrm{m} 1}(\mathrm{R} p)^{+},{ }_{3} \mathrm{p}^{+}, \mathrm{j}_{4}^{-}{ }_{5} \mathrm{R}^{0}\right\}$ в этом случае

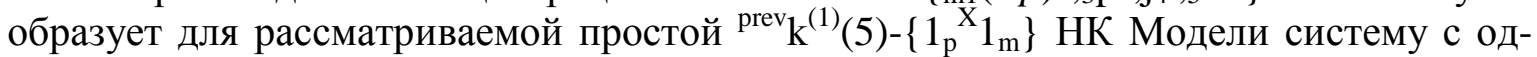
ной парой $\left\{1_{\mathrm{p}} \mathrm{X} 1_{\mathrm{m}}\right\}$ сорбирующихся компонент. Целевые компоненты, включают другой ключевой ${ }_{5} \mathrm{R}^{0}$-(нано-сайты) $5^{\text {й }}$-компонент, введенный в работах [2-6].

Новая ${ }^{\text {new }} \mathrm{k}^{(2)}-\left\{2_{\mathrm{p}}{ }^{\mathrm{X}} 2_{\mathrm{m}}\right\}$ НК Модель позволяет решить задачу дальнейшего теоретического компьютерного исследования ММП НК кинетического сорбционного процесса с включением исследования взаимодействия $\mathrm{X}_{1,2}(L, \mathrm{~T})$-концентрационных волн. Расчеты включают двух-компонентный $\left\{\mathrm{K}_{\mathrm{S}}{ }^{1} ; \mathrm{K}_{\mathrm{S}}{ }^{2}\right\}$-фактор Селективности в ЗДМ-соотношениях (I. $\left.\mathrm{K}_{1,2}\right)$ (рис. 1a-c).

В результате все исследуемые n-компоненты являются участниками 6компонентной би-функииональной ${ }^{\mathrm{new}} \mathrm{k}^{(2)}-\left\{2_{\mathrm{p}} ; 2_{\mathrm{m}}\right\}$ НК Модели, с учетом Iㄴ(II) $\}$ ММП со-маршрутов для НК сорбционной системы (см. визуальные концептуальные диаграммы на рис. $1 a$-с). Все принципиальные n-участников-компонент ММП НК кинетического процесса представлены в Таблице в таком порядке, как они здесь

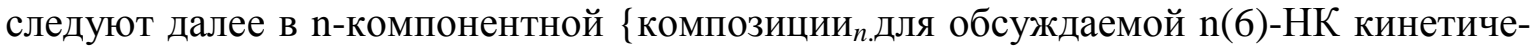
ской системы. В крайних (правом и левом) столбцах Таблицы (внизу) показаны сравнение целевых и принципиальных $\mathrm{p}, \mathrm{m}, \mathrm{R}^{0}$-компонентов новой $\left(\right.$ слева, $\left.{ }^{\mathrm{new}} \mathrm{k}^{(2)}\right)$ и предыдущей (cправa, ${ }^{\text {prev }} \mathrm{k}^{(1)}$ ) НК Моделей, используемых в моделировании ММП НК процесса

Из сравнения компонентов в крайних (левом, $\mathrm{k}^{(2)}(6)$ и правом $\left.\mathrm{k}^{(1)}(5)\right)$ столбцах демонстрирует очевидное преимущество новой ${ }^{\text {new }} \mathrm{k}^{(2)}$-Модели - фундаментальная 
возможность исследования взаимодействии двух пар (“сорбат-сорбент”) $\left\{2_{\mathrm{p}} ; 2_{\mathrm{m}}\right\}$ сорбируемых $\left\{{ }_{(3,4)} \mathrm{p}^{+} / \mathrm{m}_{(1,2)}{ }^{+}\right\}$-участников. Принципиальная возможность для двух парных принципиалов (а в предшествующей ${ }^{\mathrm{prev}} \mathrm{k}^{(1)}$ Модели присутствует только одна пара $\left\{{ }_{3} \mathrm{p}^{+} / \mathrm{m}_{1}{ }^{+}\right\}$поэтому взаимодействие сорбируемых пар- невозможно в $\left\{1_{\mathrm{p}}{ }^{\mathrm{X}} 1_{\mathrm{m}}\right\}$ НК системе.

Таблица. Сравнение свойств двух НК Моделей с различной k-вариантностью: современной ${ }^{n e w} k^{(2)}(6)$-расширенной $\quad$ (и $\quad$ предыдущей- $\quad{ }^{p r e v} k^{(1)}(5) \quad$ ), где ${ }^{\text {new }} \mathrm{k}^{(2)}(6)>{ }^{\mathrm{prev}} \mathrm{k}^{(1)}(5)$.

\begin{tabular}{|c|c|c|}
\hline${ }^{\text {new }} \mathrm{k}^{(2)}-\left\{2_{\mathrm{p}}^{\mathrm{X}} 2_{\mathrm{m}}\right\} \mathrm{HK}$ & $\left\{{ }_{\mathrm{m} 1,2}(\mathrm{pR}) ;{ }_{3,4} \mathrm{p} ; \mathrm{j}_{5}{ }_{6}{ }_{6} \mathrm{R}^{0}\right\} \mid\left\{\mathrm{ml}(\mathrm{pR}) ;{ }_{3} \mathrm{p} ; \mathrm{j}_{4} ;{ }_{5} \mathrm{R}^{0}\right\}$ & ${ }^{\mathrm{prev}^{\mathrm{k}}}{ }^{(1)}-\left\{1_{\mathrm{p}}^{\mathrm{X}} 1_{\mathrm{m}}\right\} \mathrm{HK}$ \\
\hline $\mathrm{m}(1,2)(\mathrm{R} p)^{+}$-два & $\begin{array}{c}\text { сорбирующиеся целевые компоненты- принци- } \\
\text { палы(principals) }\end{array}$ & ${ }_{\mathrm{m} 1}(\mathrm{R} p)^{+}$, один \\
\hline$(3,4) \mathrm{p}^{+}-$два & $\left(\mathrm{D}_{3.4}>0\right)$-компоненты сорбата- диффузанта; & ${ }_{3} \mathrm{p}^{+}$, oduн, $\mathrm{D}_{3}>0$ \\
\hline $\mathrm{j}_{5}^{-}\left(\mathrm{D}_{5}>0\right)$ & ко-ионы & $\mathrm{j}_{4}{ }^{-}\left(\mathrm{D}_{4}>0\right)$ \\
\hline${ }_{6} \mathrm{R}^{0}\left(\mathrm{D}_{6}=0\right)$ & $\begin{array}{c}\text { ключевой принципал (“НЧ }{ }^{0} \text {-наносайты”), под } \\
\left.\text { последним номером (6 } 6^{\text {й }} \text { или } 5^{\text {й }}, \text { где } \mathrm{D}_{6}=0\right)\end{array}$ & ${ }_{5} \mathrm{R}^{0}\left(\mathrm{D}_{5}=0\right)$ \\
\hline
\end{tabular}

Фиксированные принципиальные 1,2,6-компоненты (т.e. $\mathrm{D}_{1,2,6}=0$ ) совместно с другими диффундирующими ${ }_{(3,4)} \mathrm{p}^{+}$-принципиальными сорбат-компонентами, которые участвуют в двух парных $3 Д \mathrm{M}_{\mathrm{S}}\left(\mathrm{I}_{1,2}\right)$-реакционных соотношениях, а именно стадии “сорбции $(\mathrm{I} a-)$-десорбции $(-\mathrm{I} d)$ ” (рис. $1 a-c)$. Реакции $\left(\mathrm{I}_{1,2}\right)$ производят соответствующие селективные “трансформации масс" $\left\{3,4 \mathrm{p}^{+}+{ }_{6} \mathrm{R}^{0} \rightleftharpoons \mathrm{m}_{1,2}\right\}$ (рис. $1 a, b$ ) посредством решающего "сток-источник” ММП механизма [2-6] (рис.1a-c), который включен с помощью авторской новой развитой би-функциональной ${ }^{\text {new }} k^{(2)}(6)-\left\{2_{\mathrm{p}}{ }^{\mathrm{X}}{ }_{2}\right\}$ НК Модели, разработанной и иллюстрированной здесь на рис. $1 a$-с.

Распространяющиеся много-n(6)-компонентные $\quad\left\{\mathrm{X}_{\mathrm{n}}(\mathrm{L}, \mathrm{T})\right\}$ концентрационные волны в ММП НК кинетике сорбции для рассчитываемой $\mathrm{n}(6)-$ компонентной системы включают ММП для шести компонент-участников (перечисленных в Таблице): Решающий, ключевой ${ }_{6} \mathrm{R}^{0}$-фиксированный $6^{\text {й}}$-наносайткомпонент введен намеренно в этой публикации для описания различных $\left[{ }_{6} \mathrm{R}^{0}\right]$ концентраций $6^{\text {го }}$ компонента ("НЧ ${ }^{0}$-наносайты”). Наличие концентрации $5^{\text {го }}$ компонента $\left(\mathrm{j}_{5}{ }^{-}\right)$-ко-ионов необходимо для поддержания электро-нейтральности в ММП НК кинетической системе.

Такой обобщенный подход был ипользован ранее в авторских публикациях [2-6] для упомянутой прежде простой авторской ${ }^{p r e v} k^{(1)}(5)-\left\{1_{p}{ }_{1} 1_{m}\right\}$ HК бuфункциональной Модели с меньшей ${ }^{\operatorname{prev}} \mathrm{k}^{(1)}(5)$-вариантностью

\section{ных $X_{n}$ - волн}

Феноменологическая волновая (W)- концепция многокомпонент-

Фундаментальная феноменологическая (W)-«волновая» концепция многокомпонентных $\left\{\mathrm{X}_{\mathrm{n}}(L, \mathrm{~T})\right\}$-концентрационных волн широко используется в термодинамике неравновесных процессов во многих научных областях для теоретического описания многокомпонентного транспорта в кинетике и динамике ММП сорбционных систем.

В обширных обзорах $[2,8,9]$ представлена феноменологическая волновая Wконцепция для многокомпонентных концентрационных волн, которые имеют широкую область применения в таких областях исследования как процессы перколяции, механика жидкости, газовая динамика, теория горения , даже движения транспорта и ряда других. 
Ключевая волновая $\mathrm{W}$-концепция широко используется в теоретическом описании многих научных областей многокомпонентного переноса для различных кинетических и динамических ММП систем[2,8,9]. Все эти варианты обозреваются в хорошо известной, прекрасной монографии, посвященной теории идеальной хроматографии [8].

Используемая здесь далее феноменологическая и фундаментальная W- волновая концепция является потенциально общей и применимой ко всем фильтрационным процессам, которые могут к тому же распространяться на целую серию миграционных явлений таких как теория хроматографии, седиментация , дистилляция, электрофорез ,уличное движение и ряд других. [2,8,9]

В современных публикациях [2-6] фундаментальная феноменологическая $(\mathrm{W})$-концепция описанная выше (и указанная здесь как $\mathrm{W}$-волновая) используется в визуальных демонстрациях с помощью соответствующего современного метода визуализации волн(см. ниже “описание НКА.avi”-видео-файлов). Распространение много $(n)$-компонентных $\left\{\mathrm{X}_{\mathrm{n}}(L, \mathrm{~T})\right\}$-концентрационных волн вычисляется в современном компьютерном моделировании здесь и ранее[2-6] на основе компьютеризированного численного решения дифференциальных n-yp-ний массо-переноса (в частных производных) для ММП НК комбинированных сорбционных нелинейных систем на основе относительно простых рассмотренных здесь НК Моделей, характеризующихся соответствующими термодинамическими вариантностями : ${ }^{p r e v} \mathrm{k}^{(1)}(5)$ и ${ }^{\mathrm{new}} \mathrm{k}^{(2)}(6)$, где ${ }^{\text {new }} \mathrm{k}^{(2)}(6)>{ }^{\text {prev }} \mathrm{k}^{(1)}(5)$.

Физический смысл рассматриваемых n-компонентных $\left\{\mathrm{X}_{\mathrm{n}}(\mathrm{L}, \mathrm{T})\right\}$ распространяющихся концентрационных волн следует, очевидно, интерпретировать и обьяснять как "распространение возмущений вдоль пространственной $L$ координаты с течением Т-времени”, следуя известному определению концентрационных волн данных в фундаментальной публикации [10].

В современных авторских публикациях [2-6] фунламентальная "Wконцепция" использована в видео демонстрациях на основе соответствующего упомянутого метода визуализации посредством Научной Компьютерной "НКАнимации”, которая обозначена здесь как “НКА.avi”-видео файл (детали НКА.avi даны далее).

Запуск любого "НКА-видеофайла" демонстрирует распространение многокомпонентных $\left\{\mathrm{X}_{\mathrm{n}}(L, \mathrm{~T})\right\}$-концентрацционных волн, в НК планарной L-мембране c течением Т-времени в ММП НК кинетическом сорбционном процессе. Пример расчетного компьютерного волнового процесса на основе упомянутой расширенной бифункциональной ${ }^{\text {new }} \mathrm{k}^{(2)}(6)-\left\{2_{\mathrm{p}}{ }^{\mathrm{X}} 2_{\mathrm{m}}\right\}$ НК Модели дан здесь на рис.2А,В (ниже).

\section{Не-дифффузионные $\mathrm{X}_{1,2,6}$-концентрационные волны, распростра- няющиеся в комбинированной НК матрице и их физический смысл.}

Кинетический сорбционный ММП процесс в комбинированных НК матрицах включает два вида Массо-Переноса, т.е. парный $\left(\mathrm{I}_{1,2}\right)$-селективный ММП I]маршрут, включающий масс трансформационный механизм “сток-источник” с двух компонентным фактором селективности, т.е. $\left\{\mathrm{K}_{\mathrm{S}}{ }^{1} ; \mathrm{K}_{\mathrm{S}}{ }^{2}\right\}$-параметром Селективности вместе со вторым ММП НК $\left\{\mathrm{D}_{3-5}\right\}$-много-Диффузионным Ю-маршрутом с деталями, изображенными визуально на рис. 1a-c . Два диффундирующих (3,4) $\mathrm{p}^{+}-$ принципиальных (целевых) сорбат- компонента, а также ј5-ко-ионов вдоль многоДиффузионного $\left\{\mathrm{D}_{3-5}\right\}$ ключевым $\left.{ }_{6} \mathrm{R}^{0}\right\}$-принципиалами (Таблица) на селективном ММП Омаршруте. Все пе- 
речисленные 6-компонентов являются участниками комбинированного, нелинейного и усложненного ММП би-функционального НК кинетического процесса сорбции.

Принимая во внимание дополнительную сложность связанную с влиянием электрического поля, соотношение электронейтральности, а также учитывая фундаментальные соотношения Нернста-Планка для $\mathrm{J}_{\mathrm{p}, \mathrm{m}, \mathrm{k}, \mathrm{j}}$-потоков масс (рис. $1 a, b$ ) можно охарактеризовать ММП НК сорбционную кинетику как феноменологический усложненный многокомпонентный и нелинейный процесс, который можно охарактеризовать только на основе приближенного компьютерного моделирования с решением n-уравнений ММП для $\mathrm{J}_{\mathrm{p}, \mathrm{m}, \mathrm{k}, \mathrm{j}}$-массовых потоков (рис. $1 a, b$ ) следует характеризовать ММП НК сорбционную кинетику феноменологически как усложненный многокомпонентный и нелинейный процесс с решением только на основе обобщенного (и приближенного компьютерного решения n-уравнений материального баланса [2-6], которое обсуждается здесь вкратце. (см. рис. $1 a, b$ ).

После компьютерного решения необходимо подготовить и ввести визуальное описание многокомпонентного ММП НК сорбционной кинетики с использованием волновой $\mathrm{W}$-концепции, рассматриваемой совместно на основе метода авторской визуализации, получаемых путем численного компьютерного решения уравнений материального баланса на основе внедрения авторских би-функциональных НК Моделей.

Обработка результатов численного решения на основе авторской визуализации рассмотренная далее, приводит к картине многокомпонентных распространяющихся $\left.\mathrm{X}_{\mathrm{n}}(\mathrm{L}, \mathrm{T})\right\}$-концентрационных волн, представленных на рис. $2 \mathrm{~A}, \mathrm{~B}$ в виде «рамок-фреймов» на одной горизонтали" (представленные на рис. 2, $\mathrm{T}^{3}$ слева,> $\mathrm{T}^{2}$,середина> $\mathrm{T}^{1}$ справа; $\mathrm{T}^{1-3}$-серия). Детали авторской вмзуализации обсуждены далее.

Два параметра различных $\left\{\mathrm{K}_{\mathrm{S}}{ }^{1} ; \mathrm{K}_{\mathrm{S}}{ }^{2}\right\}$-селективностей, и парные трансформации масс целевых компонентов-участников $\left\{\left(3, \mathrm{p}^{\mathrm{X}} \mathrm{m}_{1,2}\right\}\right.$ на активных ${ }_{6} \mathrm{R}^{0}$ - нано-сайтах в прямой sorption Ia -)-и обратной desorption (If-) стадиях (см. рис. 1a-c) приводит к Вытеснительному Режиму (ВР) для принципиальных $\mathrm{X}_{1,2}$-концентрационных волн 1-(вытеснителя), и 2-(вытесняемого компонента) с учетом сорбции-десорбции $\left(\mathrm{I}_{1.2}\right)$ $\mathrm{m}(1,2)(\mathrm{R} p)^{+}$-селективных компонентов (где $\left.\mathrm{D}_{1,2}=0\right)$. Вычисляемый (моделируемый) $\mathrm{BP}$ показан на “фреймах" рис. $2 \mathrm{~A}, \mathrm{~B}\left(\mathrm{~T}^{1-3}\right.$-серии: $\mathrm{T}^{3}$, слева $>\mathrm{T}^{2}$, середина $>\mathrm{T}^{1}$, справа).

Влияние и участие $\{(3,4)$ p $\}$--принципиалов-диффузантов в селективных парных $\left(\mathrm{I}_{1,2}\right)-3 Д \mathrm{M}_{\mathrm{S}}$ реакциях (рис. $1 b$ ), принципиально сказывается на $\left\{\mathrm{X}_{1-6}\right\}$ концентрационных изменениях. Свойства диффузионной подвижности (3,4) сится в результате действия $3 Д \mathrm{M}_{\mathrm{S}}\left(\mathrm{I}_{1.2}\right)$ реакций на поведение двух не-обычных (и недиффундирующих) $\mathrm{X}_{\mathrm{m}(1,2)}(\mathrm{L}, \mathrm{T})$-концентрационных принципиальных волн с учетом не-способных к диффузии фиксированных $(\mathrm{m}(1,2) \mathrm{R} p)^{+}$-принципиалов-“комплексов" (где $\left.\mathrm{D}_{1,2}=0\right)$. ЗДМ ${ }_{\mathrm{S}}\left(\mathrm{I}_{1,2}\right)$-реакции учитыают роль сопутствующих селективных $\left\{\mathrm{K}_{\mathrm{S}}{ }^{1} ; \mathrm{K}_{\mathrm{S}}{ }^{2}\right\}-$ ЗДМ $\left(\mathrm{I}_{\mathrm{S}} \mathrm{K}_{1,2}\right)$-соотношений ( рис. $\left.1 b\right)$.

Рисунки 2А(вверху).В(внизу) ( $\mathrm{T}^{1-3}$-серии) демонстрируют поведениедвижение в горизонтальном $L$-направлении справа-налево (т.е. к $\mathrm{T}^{3}$ слева, от $\leftarrow \mathrm{T}^{1}$ справа,см. рис $2 \mathrm{~A}\left(\right.$ вверху),В(внизу) для не-обычных распространяющихся $\mathrm{X}_{1,2,6}(L, \mathrm{~T})-$ принципиальных концентрационных волн (где нет диффузионности $\mathrm{D}_{1,2,6}=0$ ), так что естественно, что распространение не-диффундирующих $\mathrm{X}_{1,2,6}(L, \mathrm{~T})$--волн определяется диффузионным распространением $\mathrm{X}_{3,4}$-концентрационных волн в НК матрице (где $\mathrm{D}_{3-5}>0$ ). Движение диффундирующих $\mathrm{X}_{3,4}$-концентрационных волн демонстрируется на рис. $2 \mathrm{~A}\left(\mathrm{X}_{3,4}\right.$-концентрационные профили изображены на рисунке тонкими линиями). 
Хроматографический визуальный BP (DD-Displacement Development)-эффект на рис. 2A,B (волновые $\mathrm{T}^{1-3}$-серии) происходит вследствии интерференциивзаимодействия посредством сорбционных $\left(\mathrm{I}_{1,2}\right) \&\left(\mathrm{I}_{1,2} \mathrm{~K}_{1,2}\right.$-соотношений для трех принципиальных $\left\{\mathrm{X}_{\mathrm{m}(1,2), 6}(\mathrm{~L}, \mathrm{~T})\right\}$-распространяющихся волн, рис. 2(A,B). Концентрационные $\left\{\mathrm{X}_{\mathrm{m}(1,2), 6}(L, \mathrm{~T})\right\}$-принципиальные сорбционные волны демонстрируют распространение необычного типа, при котором отсутствует диффузионная подвижность соответствующих 1,2,6-компонентов (т.е. $\mathrm{D}_{1,2,6}=0$ ) совместно с сопровождающим распространением диффузионных $\left\{\mathrm{X}_{(3,4) \mathrm{p}}(\mathrm{L}, \mathrm{T})\right\}$-концентрационных волн, характеризующихся диффузионно подвижными $(3,4) \mathrm{p}^{+}$-сорбат принципиаламикомпонентами (где диффузионная под-вижность $\left.\mathrm{D}_{3,4}>0\right)$ на рис. $2 \mathrm{~A}\left({ }_{(3,4)} \mathrm{p}\right.$, тонкие $3,4-$ линии в $\mathrm{T}^{1-3}$-серии; движение к $\mathrm{T}^{3} \leftarrow$ от $\left.\mathrm{T}^{1}\right)$.

Соответствующие авторские концептуальные схемы для би-функииональной ${ }^{n e w} \mathrm{k}^{(2)}(6)-\left\{2_{\mathrm{p}} \mathrm{X}_{2}\right\}$ НК Модели (рис. 1 1 -с) отчетливо демонстрируют комбинационный эффект би-функциональной ММП НК сорбционной системы, включая основной селективный механизм "сток-источник потоков масс (рис. 1с), а также два парных (I.K $\left.\mathrm{K}_{1,2}\right)-3 Д \mathrm{M}_{\mathrm{S}}$ соотношения для ММП П-маршрутов, сопровождающиеся влиянием много-диффузионного со-маршрута (U. где $\mathrm{D}_{3,4,5}>0$ ) (рис. $2 \mathrm{~A}, \mathrm{~B} ; \mathrm{T}^{1-3}$-серии).

\section{Хроматографический Вытеснительный Режим (BP-DD) и Поведе- ние Необычных $\left\{X_{1,2,6}\right\}$-Концентрационных Волн}

Селективное хроматографическое (BP-DD) взаимодействие зависит от ряда контролирующих (определяющих) факторов (см. также выше). Таким образом, интерференция распространяющихся \{селективных, $\mathrm{X}_{1,2,6}(L, T)$ и диффузионных, ${ }_{\mathrm{p}} \mathrm{X}_{3,4}$ $(L, T)\}$-принципиальных концентрационных волн для парных $\left\{2_{\mathrm{p}} \mathrm{x}_{2}\right\}$-принципиалов-

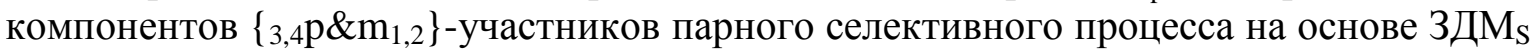
$\left(\mathrm{I}_{\mathrm{K}} \mathrm{K}_{1,2}\right)$-соотношений (рис. $1 b$ ) зависит от двух компонентного' ЗДМ $\mathrm{S}$ фактора $\left\{\mathrm{K}_{\mathrm{S}}{ }^{1} ; \mathrm{K}_{\mathrm{S}}{ }^{2}\right\}$-Селективности в (I. $\left.\mathrm{K}_{1,2}\right)$, включая также дополнительно величины входных $\left\{\mathrm{X}_{1.2}{ }^{0}\right\}$-концентраций (см. рис. $1 a$ ), и наконец от много-Диффузионного $\left\{\mathrm{D}_{3-5}\right\}$ фактора на ММП Ш со-маршруте в НК матрице (см. рис. 1a-c совместно с рис. 2, где приведена расчетная моделируемая $\mathrm{T}^{1-3}$-серия волн, обсуждаемая здесь и ранее ).

Процесс ММП НК фронтальной сорбционной кинетики с наличием $\left\{\mathrm{X}_{\mathrm{n}}\right\}$ волн (рис. $2 a-c)$ начинается, когда композиция смеси шести $(\mathrm{n}=6)$ входных $\left\{\mathrm{X}_{1-6}{ }^{0}\right\}$ концентраций, а именно $\left\{1,2\left[\mathrm{Rp}^{+}\right]^{0} ;\left[{ }_{(3,4)} \mathrm{p}^{+}\right]^{0} ;\left[\mathrm{X}_{\mathrm{j} 5}\right]^{0} ;\left[{ }_{6} \mathrm{R}^{0}\right]^{0}\right\}$ входит в ММП НК k ${ }^{(2)}(6)-$ селективную комбинированную НК MMП систему для матрицы L-мембраны (см. рис. $1 a)$ с указанной $\left\{\mathrm{X}_{1-6}{ }^{0}\right\}$-композицией на входе $\left(L_{0}=1\right)$ мембраны (см. рис. 1,2 (серия $\mathrm{T}^{1-3}$ по “движение по горизонтали”: $\mathrm{T}^{3}$,слева $>\mathrm{T}^{2}$, середина $>\mathrm{T}^{1}$, cправа).

Интенсивность хроматографического BP(DD)-эффекта определяется влиянием в основном величинами Селективности. Основную роль для интенсивности хроматографического ВP (DD)-эффекта играют величины двух компонентного $\left\{\mathrm{K}_{\mathrm{S}}{ }^{1} ; \mathrm{K}_{\mathrm{S}}{ }^{2}\right\}$-фактора селективности, которые определяют $\mathrm{X}_{1}$-“вытеснитель”, а также менее селективный вытесняемый $\left(\mathrm{K}_{\mathrm{S}}{ }^{2}\right) \mathrm{X}_{2}$-приинципиальный компонент (сплощная линия,кривая, 2).

Интенсивность BP(DD)-эффекта количественно характеризуется $\mathrm{X}_{2}{ }^{\mathrm{mx}}$-max величиной в профиле $\mathrm{X}_{2}\left(\mathrm{~L}, \mathrm{~T}^{1-3}\right)$-концентрационной вытесняемой волны (рис. 2, от $\mathrm{T}^{1}$, справа к $\mathrm{T}^{3}$, слева). Максимальная $\mathrm{X}_{2}{ }^{\mathrm{m}}$-величина отмечена черным кружком (О)на пике $\mathrm{X}_{2}{ }^{\mathrm{m}}\left(\mathrm{L}_{1-3}\right)$ для расстояний, отмеченых белыми точками $(\mathrm{O})$, изображенными на трех рис. 2A,B ( $\mathrm{T}^{1-3}$-серии, по “горизонтали”. справа (от $\left.\mathrm{T}^{1}\right)$ к $\mathrm{T}^{3}$ (налево; $\left.\mathrm{T}^{3}>\mathrm{T}^{1}\right)$.

Эффективное разделение недиффундирующих (т.е. фиксированных, т.к. $\left.\mathrm{D}_{1,2}=0\right) \quad \mathrm{X}_{\mathrm{m}(1,2)}$-принципиальных компонентов-“комплексов” зависит от контроли- 
рующих параметров, включающих дополнительно к упомянутым ранее величинам $\left\{\mathrm{D}_{3,4,5}\right\}$-диффузивностей. $\mathrm{BP}(\mathrm{DD})$-вытеснение $2^{\text {го }}$-принципиального вытесняемого компонента $\left(\mathrm{X}_{2}\right)$ определяется принципиальным компонентом-вытеснителем $\left(1^{\mathrm{M}}\right)$, влияние которого особенно эффективно на начальной стадии кинетического процесса (до T $\leq 3(\mathrm{~A})$ или 10(B)) ВР режима (DD Displacement Development) целиком изображенного во времени посредством $\mathrm{T}^{1-3}$-серии на трех рис. 2А,В (слева, середина, справа; $\left.\mathrm{T}^{1,2} \leq 12\right)$.

A. ${ }_{\mathrm{A}} \mathrm{T}^{1-3}=12>7>3$

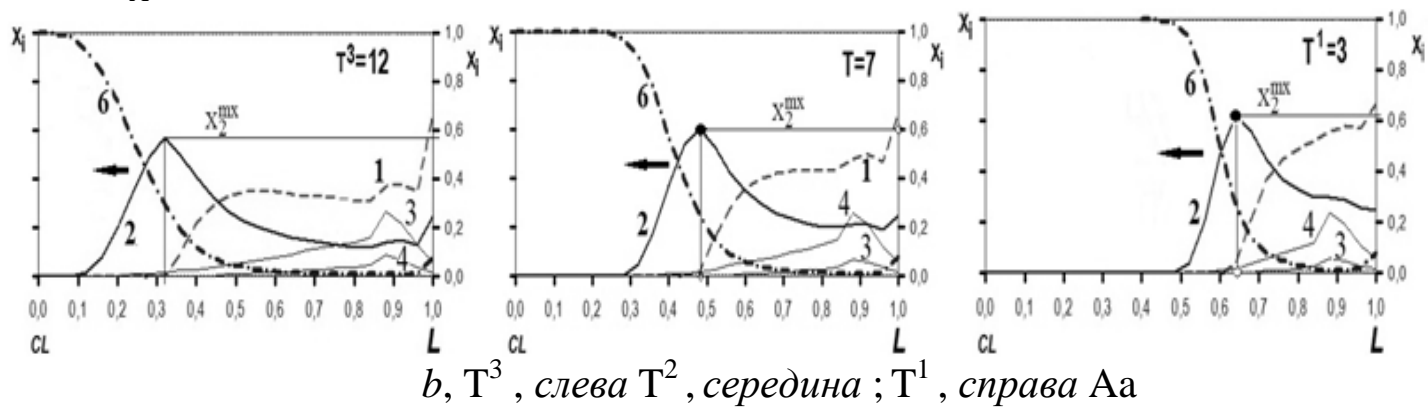

B. ${ }_{\mathrm{B}} \mathrm{T}^{1-3}=18>12>6$
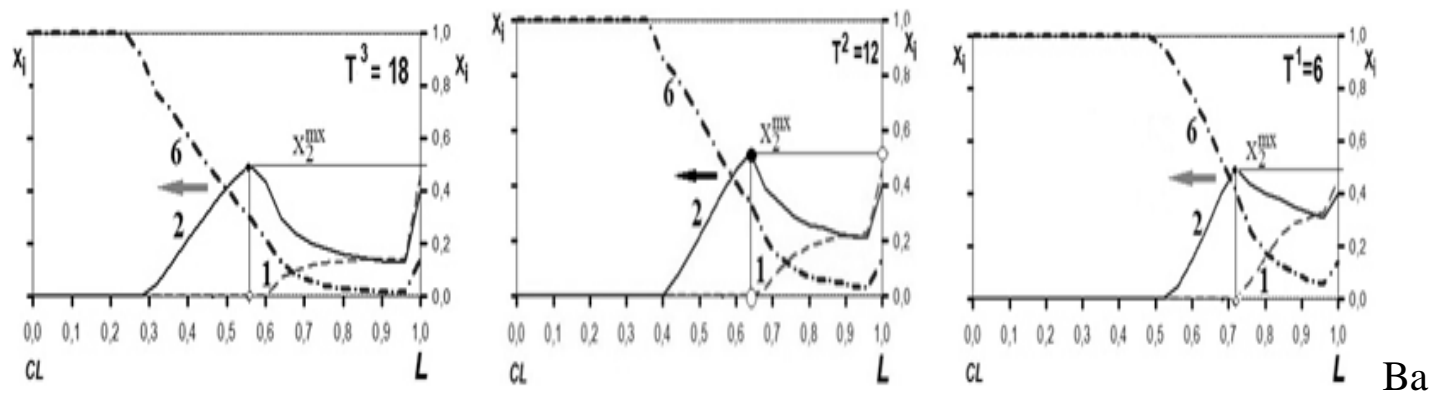

Рис. 2А(вверху),В (внизу). “Эффективное вытеснение $\mathrm{X}_{1,2}$-концентрационных волн (1-вытеснитель, пунктир; 2-вытесняемый, сплошная) через HK L-мембрану (A; В - движение 1-6 компонентов "по горизонтали "налево $\left(\mathrm{T}^{3}\right) \leftarrow$ справа $\left(\mathrm{T}^{1}\right)$ ",) для двух различных вариантов вытеснения, А,В (к $\mathrm{T}^{3}$ от $\mathrm{T}^{1}$ (см. А ; В),где Селективности $\mathrm{K}_{\mathrm{SA}}{ }^{1}(400)>\mathrm{K}_{\mathrm{SB}}^{1}(320)$. Два А,В-варианта селективного ММП процесса ( ${ }^{3}$, слева $>\mathrm{T}^{2}$,середина $>\mathrm{T}^{1}$, справа $)$. "Рисунки-фреймы" для $\left\{\mathrm{X}_{1-6}\left(L, \mathrm{~T}^{1-3}\right)\right\}$ распространяющихся волн (к $\mathrm{T}^{3}$, слева >от $\mathrm{T}^{1}$, справа). Диффузивности $\left\{\mathrm{D}_{3}=0,1 ; \mathrm{D}_{4}=0,055 ; \mathrm{D}_{5}=0,02\right\}^{\mathrm{A}, \mathrm{B}}$ и Селективности: $\left\{\mathrm{K}_{\mathrm{SA}^{1}}(400) ; \mathrm{K}_{\mathrm{SA}^{2}}{ }^{2}(50)\right\}$ или $\left\{\mathrm{K}_{\mathrm{SB}}{ }^{1}(320) ; \mathrm{K}_{\mathrm{SB}}{ }^{2}(40)\right\} . L, \mathrm{~T}, \mathrm{~K}_{\mathrm{S}}^{1,2}, \mathrm{D}_{3-5}, \mathrm{X}_{\mathrm{n}}$ (безразмерные).

Очевидно, что вытеснение $\mathrm{X}_{2}$-волны проходит более эффективно $\left({ }_{\mathrm{A}} \mathrm{X}_{2}{ }^{\mathrm{mx}}>\right.$ ${ }_{\mathrm{B}} \mathrm{X}_{2}{ }^{\mathrm{mx}}$ ) для варианта $\mathrm{A}\left(\right.$ вверху), для которого $\mathrm{K}_{\mathrm{SA}}{ }^{1}(400)>\mathrm{K}_{\mathrm{SB}}{ }^{1}(320)$. Влияние величин диффузионных $\mathrm{D}_{3-5}$-коэффициентов будет рассмотрено в подготовляемых публикациях.

\section{Трансформация матричных данных компьютерного моделирова- ния и Визуализация на основе много( $(n=6)$-цветных “НКА.avi” видео- файлов}

Финальные результаты численного моделирования (полученные в результате авторского компьютерного моделирования ММП НК сорбционных систем) представляются набором численных математических $\|\mathrm{A}\|\left(\mathrm{T}^{i}\right)$-матриц для соответствующих текущих дискретных $\mathrm{T}^{i}$-моментов времени. Матрицы расположены в последо- 
вательности увеличения дискретных $\mathrm{T}^{i}$ - величин времени $(i=1,2, \ldots)$. Любой численный $\mathrm{A}_{\mathrm{nj}}\left(\mathrm{T}^{i}\right)$-элемент $\|\mathrm{A}\|\left(\mathrm{T}^{i}\right)$-матрицы (для данного $\mathrm{T}^{i}$-момента) описывает $\mathrm{n}$ волновые $\left\{\mathrm{X}_{\mathrm{n} j}\right\}$-концентрации, где первый n-индекс (номер столбца $\|\mathrm{A}\|$-матрицы) соответствует номеру $\mathrm{n}$ - компонента $(1 \leq \mathrm{n} \leq 6)$, а второй бегущий индекс (на $\mathrm{j}^{\mathrm{n}}$ столбце $\|\mathrm{A}\|$-матрицы) соответствует $\mathrm{L}_{j}$-положению $\left(0 \leq \mathrm{L}_{j} \leq 1\right)$ данной $\mathrm{X}_{\mathrm{n} j}$ концентрации в n-волне, где следует записать $\mathrm{A}_{\mathrm{n} j}\left(\mathrm{~T}^{i}\right)=\mathrm{X}_{\mathrm{n}}\left(\mathrm{L}_{j}, \mathrm{~T}^{i}\right)$.

Очевидно, что такой большой численный набор математических $\|\mathrm{A}\|\left(\mathrm{T}^{i}\right)$ матриц описывает здесь финальные результаты компьютерного моделирования для НК ММП процесса, которые в таком виде невозможно предлагать для восприятия научной аудиторией.

Поэтому совершенно естественно, что абсолютно необходимо провести последующую обработку полученных численных результатов авторского моделирования. Для этой цели здесь предлагается современный метод визуализации, описываемый далее как результирующие НКА-Научные Компьютерные Анимации (или HКA.avi-видео-файлы, см. например “картинки-фреймы” на рис. $2 \mathrm{~A}, \mathrm{~B}\left(\mathrm{~T}^{1-3}\right.$-серии). Эти фреймы представляют собой отдельные “кадры” авторских НКАнимаций [1113]

Визуальные примеры много(n)-компонентных $\left\{\mathrm{X}_{\mathrm{n}}\left(\mathrm{L}, \mathrm{T}^{i}\right)\right\}$ - концентрационных волн представлены визуально во “фреймах" на рис. $2 \mathrm{~A}, \mathrm{~B}\left(\mathrm{~T}^{1-3}\right.$ серии, “по горизонтали” ), упомянутых визуальных трансформаций с тремя кадрами из численного расчетного набора $\|\mathrm{A}\|\left(\mathrm{T}^{1-3}\right)$-матриц, содержащих результаты рассматриваемого компьютерного моделирования (на рис. $2, \mathrm{~T}^{i}$-серии, $i=1,2,3$ ).

Таким образом, численные данные, полученные здесь компьютерным ММП НК моделированием преобразуются затем с помощью компьютерной визуализации разработанной ранее и представляемой в течение десятка лет на международных [1113], а также и всероссийских конференциях довольно сложного нелинейного много(n)-компонентного динамического и ММП НК кинетического сорбционных процессов с помощью НКА.avi компьютерных Анимации названных здесь компьютерными НКА.avi” видео-файлами [2-6,11-13].

Рассмотренный авторский метод визуализации выражает финальные численные результаты посредством визуальных много-цветных "HKA(SCA).avi" видеофайлов. Метод используется в течение более 15 лет для много(n)-компонентных $\left\{\mathrm{X}_{\mathrm{n}}\left(\mathrm{L}_{j} ; \mathrm{T}^{i}\right)\right\}$-распространяющихся волн. Распространение концентрационных $\left\{\mathrm{X}_{\mathrm{n}}\left(\mathrm{L}_{j} ; \mathrm{T}^{i}\right)\right\}$-волн визуально и наглядно представлено здесь в $\mathrm{T}^{1-3}$ сериях рис. 2 (см. “фреймы” на $\mathrm{T}^{1-3}$-сериях)[2-6,11-13]. (см. рис. $2,\left\{\mathrm{X}_{\mathrm{n}}\left(L ; \mathrm{T}^{i}\right)\right\}$-концентрационные волны для $\mathrm{T}^{1-3}$-серий), где наглядные примеры показаны как “многоцветные” концентрационные профили $\left\{\mathrm{X}_{1-6}\left(L, \mathrm{~T}^{i}\right)\right\}$-распределения из HKA(SCA).avi-анимаций реализованы посредством многоцветных "НКА.avi” видео-файлов с частным представлением посредством отдельных “картинок-фреймов” для последовательности различных моментов $\mathrm{T}^{i}$-времени (рис. 2,A;B, частные $\mathrm{T}^{1-3}$-серии). Фреймы включают часть $\left\{\mathrm{X}_{\mathrm{n}}\left(\mathrm{L}, \mathrm{T}^{i}\right)\right\}$ - концентрационных волн, упорядоченных в "НКА.avi" видео-файлах для дискретных $\mathrm{T}^{i}$-моментов в течение ММП НК сорбционного кинетического процесса, полученного здесь на основе разработанного компьютерного моделирования.

Каждый "НКА.avi” видеофайл собирается при визуализации последовательно из отдельных многоцветных “картинок-фреймов"(примеры на рис.2), вычисляемых (см. выше упомянутые $\|\mathrm{A}\|$-«матрицы» в течение созданного компьютерного моделирования.

“Картинки-фреймы” $\left\{\mathrm{X}_{(1, .6)}\left(\mathrm{L}, \mathrm{T}^{i}\right)\right\}$ на рис.2 располагаются в порядке увеличения текущего дискретного $\mathrm{T}^{i}$-времени $\left(\mathrm{T}^{i}, i=1,2,3\right)$ с дискретными временными $\mathrm{dT}$ шагами. Каждый “много-ияветный $\left\{\mathrm{X}_{\mathrm{n}}\left(\mathrm{L}, \mathrm{T}^{i}\right)\right\}$-“видеокадр” (рис.. $\left.2, \mathrm{~T}^{i}, i=1,2,3\right)$ подго- 
товлен предварительно как визуальный в виде трех различных “i-образцов-фреймов” для "компьютеризированных “НКА.avi” видео-файлов.

Исполнение компьютерных 'НКА.avi' видео файлов использовалось многократно в течение десятка лет во многих устных авторских презентациях для иллюстрации процессов ММП сорбционной НК-кинетики [2-6,13] а также и динамики [1112]), например в ряде международных и общероссийских конференций, в особенности, в регулярных периодических (1 раз в 4-е года) “IEx 2004-2008-20012” конференциях в Кембридже (Cambridge, UK) [11-13].

Для рассматриваемой публикации подготовленные авторские компьютерные “НКА.avi" видео-файлы демонстрируют (в подготовленных авторских устных презентациях) непрерывное движение $\left\{\mathrm{X}_{\mathrm{n}}(L, \mathrm{~T})\right\}$-концентрационных волн (при произвольных малых шагах $\mathrm{dT}=\mathrm{T}^{(\mathrm{i}+1)}-\mathrm{T}^{\mathrm{i}}$ ) в хроматографическом Вытеснительном Режиме (Displacement Development) в течение анимационного распространения многоцветных $\left\{\mathrm{X}_{\mathrm{n}}\left(\mathrm{L}, \mathrm{T}^{i}\right)\right\}$-концентрационных волн в течение безразмерного $\mathrm{T}^{\mathrm{i}}$-времени $(i=1,2, \ldots)$, как на рис. $2 \mathrm{~A}, \mathrm{~B}$.

"Многоцветные "НКА.avi" видео-файлы, созданные для ММП НК сорбционных кинетических и динамических процессов, использовались много раз автором статьи в визуальных легко воспринимаемых компьютерных презентациях для ряда различных научных упомянутых аудиторий.[2-6,11-13]

\section{Заключение}

Применение фундаментальных концепций неравновесной термодинамики с включением разработанной новой расширенной ${ }^{n e w} k^{(2)}(6)-\left\{2_{p}{ }^{\mathrm{x}} 2_{\mathrm{m}}\right\} \quad$ бuфункциональной НК Модели (c $\left\{2_{\mathrm{p}} \mathrm{x}_{\mathrm{m}}\right\}=\left\{\mathrm{z}_{3,4} \mathrm{p}^{\mathrm{x}} \mathrm{m}_{1,2}\right\}$-принципиальными компонентами в ММП НК процессе) дает ранее неизвестные теоретические результаты (см. пп.1-7 ниже). Результаты вычисляются численным компьютерным моделированием на основе феноменологических n-уравнений баланса масс (в частных производных), описывающих ММП для сорбционной кинетики внутри современных сорбционных НК материалов.

Полученные результаты компьютерного моделирования выражены посредством много-n(6)-компонентных распространяющихся $\left\{\mathrm{X}_{\mathrm{n}}(L, \mathrm{~T})\right\}$-концентрационных волн, а затем визуализированных компьютерных "НКА.avi" многоцветных видеофайлов-НКАнимаций. Финальные результаты могут быть продемонстрированы, видео-кадрами (как альтернатива НКАнимациям в устных презентациях на конференциях) или “изображениями-фреймами” (как на рис. $2 \mathrm{~A}, \mathrm{~B}\left(\mathrm{~T}^{3}\right.$, слева $>\mathrm{T}^{2}$, середина $>\mathrm{T}^{1}$, справа).

Демонстрация результатов движения многокомпонентных $\left\{\mathrm{X}_{\mathrm{n}}\right\}$-волн НКАнимациями адекватно воспринимается научной аудиторией на семинарах и конференциях.

Краткие Основные Выводы (Highlights)

N1. Компьютерное моделирование на основе численных решений $\mathrm{n}(6)$ уравнений баланса масс для разных вариантов включает разработанные и расширенные би-функциональные $\mathrm{k}^{(2)}(6)-$-Модели Нано-Композитов (НК) (Рис.1).

N2. Компьютерные результаты моделирования приводят к интерференциивзаимодействию много-n(6)-компонентных распространяющихся $\quad\left\{\mathrm{X}_{\mathrm{n}}(L, \mathrm{~T})\right\}$ концентрационных волн в планарных HK L-мембранах (Рис. 2A.B).

N3. Феноменологическая волновая $\mathrm{W}$-концепция расширена на сорбционные $\mathrm{M}(\mathrm{n}=6) \mathrm{MП} \mathrm{явления} \mathrm{с} \mathrm{взаимодействием} \mathrm{сорбирующихся} \mathrm{принципиальных} \mathrm{компонен-}$ тов в би-функциональныхх планарных L- мембранах НК. 
N4. Созданные Визуальные компьютерные НКАнимации показывают очевидно интерференцию $\left\{\mathrm{X}_{\mathrm{n}}\right\}$-концентраионных волн с сильной зависимостью от $\mathrm{K}_{\mathrm{S}}{ }^{1}$ селективности 1-вытеснителя для парных $\left\{2_{\mathrm{p}}^{\mathrm{X}}{ }_{2 \mathrm{~m}}\right\}=\left\{3,4 \mathrm{p} \& \mathrm{~m}_{1,2}\right\}$-принципиальных компонентов, сорбирующихся на ${ }_{6} \mathrm{R}^{0}$-“нано-сайтах”.

N5. Результаты компьютерного моделирования и НКАнимации для Визуализации показывают интерференцию необычных $\left\{\mathrm{X}_{1,2,6}(\mathrm{~L}, \mathrm{~T})\right\}$-волн принципиальных 1,2,6-компонентов в НК с использованием феноменологической волновой (W)концепции.

N6. Диффузионно не-подвижные $\left(\mathrm{D}_{1,2,6}=0\right)$ и не-обычные $\left\{\mathrm{X}_{1,2,6}(\mathrm{~L}, \mathrm{~T})\right\}$ концентрационные волны приобретают подвижность через HK L-мембрану, благодаря трансформации масс при ЗДМ $\mathrm{S}$ сорбции-десорбции $\left(\mathrm{I}_{1,2}\right)$ на активных ${ }_{6} \mathrm{R}^{0}$-“наносайтах".

N7. Поведение упомянутых не-обычных, не-диффузионных $\left(\mathrm{D}_{1,2,6}=0\right)\left\{\mathrm{X}_{1,2,6}\right.$ $(\mathrm{L}, \mathrm{T})\}$ - концентрационных волн приводит к вытеснительному режиму (DD) для $\left\{\mathrm{X}_{1,2}\right\}$-волн, где 1- вытеснитель, а 2-вытесняемый компоненты в смешанной HK Lматрице.

\section{Список литературы/References}

1. Khaaze R., Termodinamika of Neravnovesnykh protsessov, Gl.2;4, M., Mir, 1967, 544 p.

2. Kalinitchev A.I., NanoTechnol Rev (NTREV). Special issue (N5) "NanoTechnology: from Convergence to Divergence”, DeGruyter Ed.3(5): 2014, pp.467-498 (. $\mathrm{http} / / /$ degruyter.com/new/ntrev2014.3 issuefiles. doi:10.1515/ntrev-2014-0007. Publ. on line 8.10, and Printed 14.10. 2014(Ed. Ja S. Lee).

3. Kalinitchev A.I., Prot. Met.\&Phys. Chem. Surf. (Springer Ed.), 2013, Vol. 49(6), pp.627638. doi:10.1134/S2070205113060051. $\mathrm{http} / / / \mathrm{www}$ springerlink. com/ openurl.asp?genre $=$ article $\&$ ID.

4. Kalinitchev A.I., Advances in Nanoparticles, AnP. 2(2), 2013, pp.1-13, Sci.Res.Publ.: SCIRP. E-Journ. doi:10.4236/ anp.2013.22028 (Site AnP:http:// www.scirp. org/ journal/anp/). ID: 31894.

5. Kalinichev A.I., Sorbtsionnye i khromatograficheskie protsessy, 2013, Vol. 13, No4, pp. 413-428.

6. Kalinitchev A., Sorbtsionnye i khromatograficheskie protsessy, 2016, Vol. 16, No 6, pp. 748-768.

Калиничев Анатолий Иванович - д.х.н. (физ. химия), гл. научный сотрудник Института физ химии и электрохимии им. акад. А.Н. Фрумкина РАН, Москва.
7. Kravchenko T.A., Polyanskiy L.N., Kalinichev A.I., Konev D.V., Nano Kompozity Metall -IonoObmennik. M., "Nauka", 2009, 390 ps. (Monografiya).

8. Helfferich F., Klein G., Multicomponent Chromatography. Theory of Interference, New York, M. Dekker Inc., 1970, 360 p.

9. Kalinitchev A., J. Rus. Chem. Reviews, 1996, Vol. 65, pp. 95-115. (English) doi:10.1070/RC1996v065n02A BEH000201.

10. Whitham G.: Linear and Nonlinear waves, Wiley. NY, 1974, 636 p.

11. Kalinitchev A.I., Hoell W.H., , Ion Exchange Technology for Today and Tomorrow, Cox M., Ed., Soc. Chem.Ind. (SCI) London, 2004, pp.53-58 (Extend. Thes., pp. 349356). www. soci.org.

12. Kalinitchev A.I., Hoell W.H., Recent Advances in IEx Theory\&Practice, Cox M., Ed. Soc.Chem.Ind. (SCI), London, 2008, pp.85-93, (www.soci.org.).

13. Kalinitchev A.I., In IEX2012 Cox M.,Ed., Soc. of Chem. Ind., London, S. Fundam.( El.Book). (Thesis: p. 123-124) . 2012, pp. 1-18.

Kalinitchev Anatoliy I. - Doctor Habilitat (Phys. Chem.), principal investigator, Institute for Phys Chemistry and ElectroChemistry named after acad. A.N. Frumkin. RAS (Moscow). E-mail: kalina@phyche.ac.ru 